\title{
Centralized Management for Efficient Control of Network Devices
}

\author{
D.Vimala, S. Sangeetha, B. Sundar Raj
}

\begin{abstract}
Computer networking is plays vital role in everyday business opportunity, network administrator and engineers has responsibility to keep network infrastructure of organization 24/7. The more up time of network the more business operates smoothly, network downtime may cause several problems in business, imagine what would happen if network of stock exchange is goes down for one are two minutes it would cause loss of million dollars. Management of network infrastructure and networking devices is quite daunting task for network engineers. In this paper we described the methods and technologies used for management and controlling of network devices. Network infrastructure management is big task for any organization. Complexity of network device management is based on the size and design of network. In recent time new technologies are emerging in management of network infrastructure like software defined networking we reviewed $S D N$ pros and cons.
\end{abstract}

Keywords : Software defined networking, Management, Routing, Open flow protocol.

\section{INTRODUCTION}

Management of network resources and data flow is become more complex in today's network. Companies invest big sum of amount in network infrastructure management, some of networking industries provide lot of services for network management. In order to manage network devices and infrastructure services network engineers and administrator have to possess lot of skill set, a company may use network devices like router, switch, firewall from different manufactures which means it has various network operating system depends on vendor of network device vendor, so the engineer Programming Defined Networking (SDN) is another methodology in systems administration Technology, intended to make significant level reflections over which equipment and programming foundation can be worked to help new distributed computing applications. SDN is likewise alluded to as programmable system, since it confines control plane from information plane and gives a free and unified unit to control the network. Central control over the network provides lot of benefits.

System mechanization is the way toward computerizing the arrangement, the board, testing, sending, and activities of physical and virtual gadgets inside a system. Consistently system errands and capacities are performed consequently. Utilizing a mix of equipment and programming based arrangements, enormous associations, specialist co-ops, and

Revised Manuscript Received on December 11, 2019.

* Correspondence Author

D.Vimala *, Department of CSE, Bharath Institute of Higher Education and Research, Chennai, Tamilnadu, India.

S. Sangeetha, Department of CSE, Bharath Institute of Higher Education and Research, Chennai, Tamilnadu, India.

B. Sundar Raj, Department of CSE, Bharath Institute of Higher Education and Research, Chennai, Tamilnadu, India. endeavors can execute organize computerization to control and oversee tedious procedures and improve system administration accessibility.

\section{PURPOSE OF TASK}

The purpose of this task is to identify various technologies used in networks for management and dataflow control and analyse advantages and disadvantages and other issues in network infrastructure. New technologies are being introduced in computer networks.

These technologies need to be researched and testing for consistency and efficiency of its operation.

These technologies may benefit a lot to business but these benefits must be analysed in various business tiers respective to cost, interoperability, portability and efficiency. Small scale industries find difficulties to moving into new technologies because of cost and portability.

\section{ISSUES IN NETWORK MANAGEMENT}

There are several complexities and issues in management of network devices. If you want to change the operating status of network. In order to configure device like router, switch and other network devices you have to configure them via CLI or telnet or SSH. The following are common challenges
1.

2.

3.

4.
Utilization of assets

Network administration

Error administration

Traffic administration
Resource utilization is one of the commonly known issues in network management, to achieve maximum throughput resources have to be utilized in efficient way. Error management is another factor of network management.

Network administrators have to analyse logs on daily basis to identify those errors and implement solutions based on error.

\section{STUDY OF SOFTWARE DEFINED NETWORKING}

Structuring and overseeing systems frameworks become a difficult errand due to the significant level of multifaceted nature associated with system plan. The tight coupling between a system's information and control plane gets an ascent different difficulties to the board and development of system. System architects should be physically change their elevated level organizations arrange approaches into low-level design directions of specific gadget,

Published By:

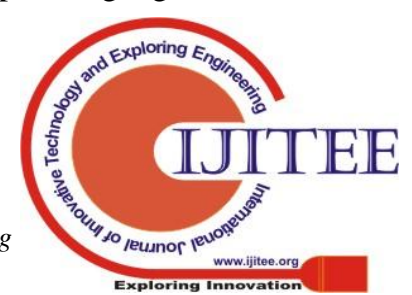




\section{Centralized Management for Efficient Control of Network Devices}

process which for complex systems can be profoundly testing and blunder inclined. Adding new usefulness to the current arrange framework, similar to interruption recognition systems(IDS), load balancers and different frameworks generally requires messing with the system's foundation and directly affects its rationale on account of progress, while sending new conventions could be a very moderate procedure and it might requesting long stretches of study and testing for network administrators.

Management of traffic is one of the daunting task of network management, complexity of traffic is based on design and size of network. Traffic management

includes classification of traffic, type of service, prioritizing traffic based on type of data.

Including new projects or including new strategies require overseer arranging various individual gadgets which will expect director to have working learning of gadget working framework (OS) and setup techniques. For some situation single system may have distinctive merchant items that makes chairman need to know about different seller items. As your system scales, it turned out to be an ever increasing number of rigid as an ever increasing number of gadgets must be designed for each change.

As referenced above to access into gadget we have to utilize CLI - direction line interface or SNMP or different access techniques and arrange control plane so as to impact new conduct in sending plane.

to guarantee its working and interoperability among the usage gave by different framework merchants.

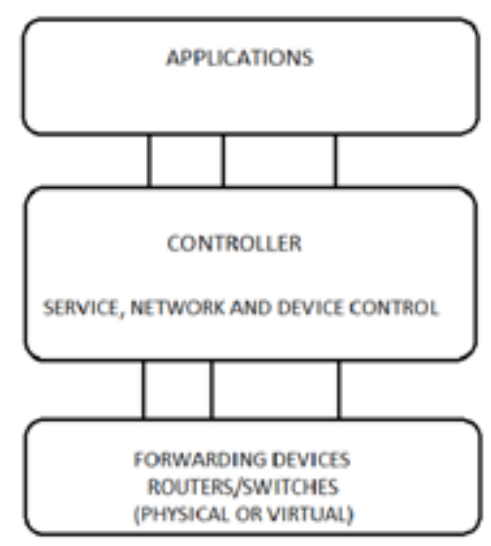

Fig. 1. SDN Approach

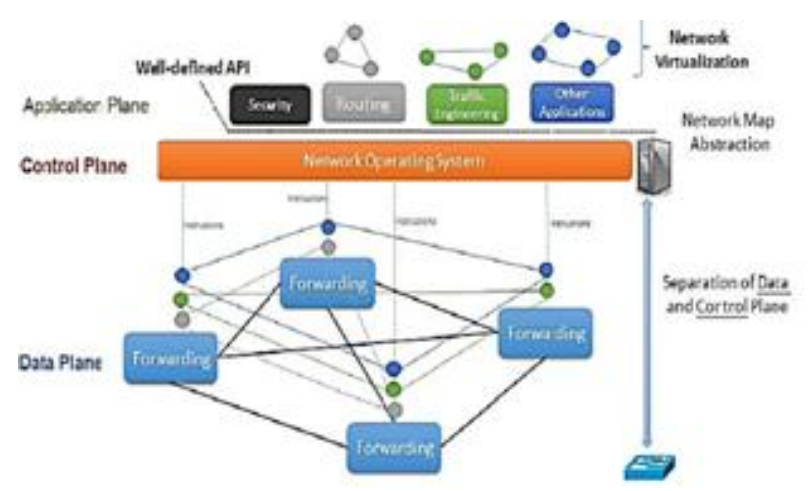

Fig. 2. Software defined network approach

\section{OPENFLOW PROTOCOL}

Following the SDN rule of decoupling the control and information planes, open Flow gives an institutionalized method for overseeing traffic in switches and of trading data between the switches and the controller, as Figure 2 represents. The Open Flow switch is made out of two sensible parts. The principal part contains at least one stream tables answerable for keeping up the data required by the switch so as to advance bundles. The subsequent part is an Open Flow customer, which is basically a straightforward API permitting the correspondence of the switch with the controller. The stream tables comprise of stream sections, every one of which characterizes a lot of guidelines deciding how the bundles having a place The pinnacle of system programmability has been advanced in past years as a way to change this circumstance by advancing new innovations in system the board and the sending of system benefits through programmability of the fundamental system substances utilizing a portion of the open system application programming interface(API). This takes us to new dependable and adaptable PC system ready to work as indicated by the client's needs in an immediate relationship to how programming dialects are being utilized to reconstruct PC frameworks so as to play out an assignments without the requirement for constant difference in the current equipment stage. Programmable system is a generally new time of a system programmability which changes how systems are structured and oversaw by presenting a reflection that decouples the control from the information plane, as outlined in Figure 1. In this strategy a control program, alluded as the focal SDN controller, has a whole perspective all in all system and is liable for the creation choices over its capacities, while the equipment is essentially liable for conveyance bundles into their goal according to the controller's directions, commonly a lot of parcel taking care of principles. Programmable system engineering is strikingly adaptable it could work with different sorts of switches and at various layers of convention.

Controller of SDN and switches can be actualized for Ethernet switches, Internet switches, transport exchanging, or application layer exchanging and directing. Programming characterized systems depends on the normal usefulness found on systems administration gadgets, which basically include sending parcels dependent on some type of stream definition. There is another significant convention that utilized by controller of to connect with gadget that is convention called open Flow.

In the traditional networking architecture, each networking device would require individual low-level configuration by the network engineer in order to operate properly as per requirements. Moreover, since each and every device in network targets a different networking technology, it would have its own specific configuration and management requirements, meaning that extra effort would be required by the administrators to make the whole network operate as required. 
On the other side, with the logically centralized control of software defined network, the administrator would not have to worry about low level details. Instead, the network management would be performed by defining a proper high level policy, leaving the network operating system responsible for communicating with and configuring the operation of network devices.

Having discussed the general concepts behind the controller of SDN, the following subsections take a closer look at specific design decisions and implementation choices made at this core component that can prove to be critical for the overall efficiency, performance and scalability of the network.

\section{SDN CONTROLLDRS}

As effectively plotted over, one of the centre thoughts of the product characterized organize theory is the presence of a system working framework set between the application layer and the system foundation. This system working framework is answerable for overseeing and organizing the assets of the entire system and for uncovering a unique bound together perspective on all parts to the applications executed over it. This thought is practically equivalent to the one followed in a PC framework, where the OS of system gadgets lies between client space and the equipment and is answerable for dealing with the equipment assets and giving regular administrations to programs for client. Essentially, organize engineers,

The logically centralized control and the generalized network abstraction it offers make the SDN model applicable to a wider range of applications and heterogeneous network technologies compared to the conventional networking paradigm. For instance, consider a heterogeneous environment composed of a fixed and a wireless network comprised of a large number of related network devices (routers, switches, wireless access points, middle boxes, etc.).

With that specific stream will be overseen by the switch (i.e., how they will be handled and sent). Every section in the stream table has three fields: (I) a bundle header characterizing the stream, (ii) an activity deciding how the parcel ought to be handled, and (iii) insights, which monitor data like the quantity of bundles and bytes of each stream and the time since a parcel of the stream was last sent. When a bundle lands at the Open Flow switch, its header is analyzed, and the parcel is coordinated to the stream that has the most comparable parcel header field. In the event that a coordinating stream is discovered, the activity characterized in the activity field is performed.

These activities incorporate the sending of the bundle to a specific port so as to be steered through the system, the sending of the parcel so as to be inspected by the controller, or the dismissal of the bundle. On the off chance that the bundle can't be coordinated to any stream, it is treated by the activity characterized in a table-miss stream section.

The exchange of information between the switch and the controller happens by sending messages through a secure channel in a standardized way defined by the Open Flow protocol. This way, the controller can manipulate the flows found in the flow table of the switch (i.e., add, update, or delete a flow entry) either Main challenges in SDN include:
- Security threats

- Attack on control plane

- Attack on vulnerabilities on switches

- Attack on controllers

- Attack on administrative stations

- Lack of trusted sources.

\section{VARIOUS CHALLENGE IN SDN}

Main challenges in SDN include:

- Security threats

- Attack on control plane

- Attack on vulnerabilities on switches

- Attack on controllers

- Attack on administrative stations

- $\quad$ Lack of trusted sources.

They can be reduced to some extent by redundancy (of devices and applications), same application to run on different controllers, self-correction and healing mechanisms and dynamic device association to another controller in case of a failure.

\section{PROPOSED SYSTEM}

Our system is web based application for network management. Unlike SDN we are not decoupling control plane from data plane, each node in a network can independently work and make decisions based on individual configuration of control plane.

Centralized management server is used for configuration and managing each and every resource on network. Only management of network is centralized control of network remain in individual nodes. In our solution automation of network is part of the framework, by using this automation module we can automate regular tasks that performed in network, this will reduce lot of overhead of network management. Tasks like backing up configuration, routine check of logs and etc. Fig. 4 is architecture of our system. Advantages of our application:
Centralized management
Distributed control
Automation of tasks
Notification of events
Ease of management 


\section{Centralized Management for Efficient Control of Network Devices}

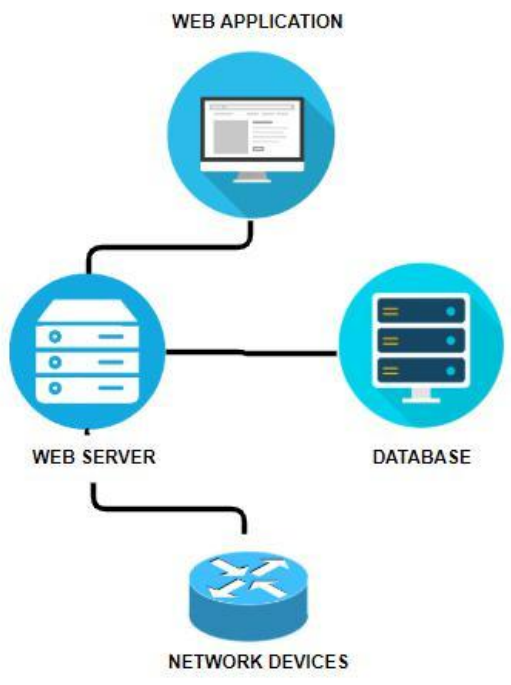

Fig. 4. Network management application architecture This application contains four primary modules illustrated in fig 4.1. It is developed by layered approach so that makes made isolation of components which makes ease of management and error isolation.

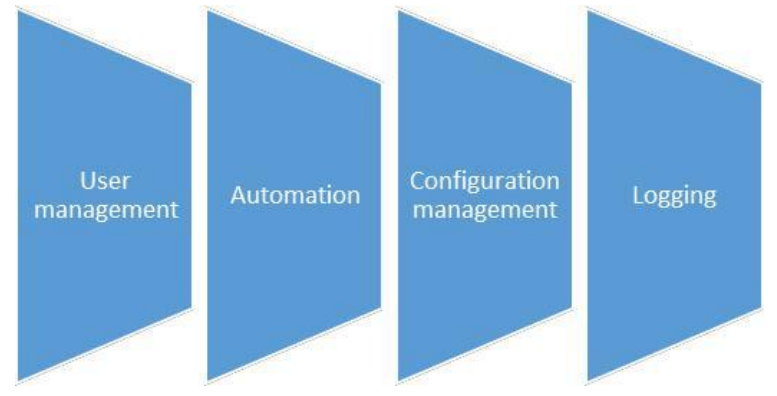

Fig. 4.1. Modules of network manager

\section{A. User Management}

User management module deals with all kind of actions related to users, such as Adding user to application, user privilege, forgot password, reset password. Only super user can add other admin users, random password generator generate password while creation of user and send those credentials over email.

\section{B. Automation}

Automation module is major part of this application. Tasks that are repeatedly performed by network administrators are automated and specified tasks are performed based on the respective time configured by user. Task includes configuration backup, log analysis, performance analysis.

\section{Logging}

Logging module create log file for each and every event in application and network devices. It can be used for if there is any inconsistency in management application and network devices.

\section{CONCLUSION}

In this paper we mentioned various technologies used in computer networks and researched issues in network management and control, also discussed about emerging networking solution software defined networking and openflow protocol its advantages and disadvantages. We also proposed our solution for management for network devices. This module manages operations related to all network device configurations. All device configurations are stored in server, operations are checking integrity of configurations, revert back consistent configuration file to device.

\section{REFERENCES}

1. Kumaravel A., Meetei O.N.,An application of non-uniform cellular automata for efficient cryptography,2013 IEEE Conference on Information and Communication Technologies, ICT 2013,V-,I-,PP-1200-1205,Y-2013

2. Kumarave A., Rangarajan K.,Routing alogrithm over semi-regular tessellations,2013 IEEE Conference on Information and Communication Technologies, ICT 2013,V-,I-,PP-1180-1184,Y-2013

3. Dutta P., Kumaravel A.,A novel approach to trust based identification of leaders in social networks, Indian Journal of Science and Technology,V-9,I-10,PP--,Y-2016

4. Kumaravel A., Dutta P.,Application of Pca for context selection for collaborative filtering,Middle - East Journal of Scientific Research,V-20,I-1,PP-88-93,Y-2014

5. Kumaravel A., Rangarajan K.,Constructing an automaton for exploring dynamic labyrinths,2012 International Conference on Radar, Communication and Computing, ICRCC 2012,V-,I-,PP-161-165,Y-2012

6. Kumaravel A.,Comparison of two multi-classification approaches for detecting network attacks, World Applied Sciences Journal,V-27,I-11,PP-1461-1465,Y-2013

7. Tariq J., Kumaravel A.,Construction of cellular automata over hexagonal and triangular tessellations for path planning of multi-robots,2016 IEEE International Conference on Computational Intelligence and Computing Research, ICCIC 2016,V-,I-,PP--,Y-2017

8. Sudha M., Kumaravel A.,Analysis and measurement of wave guides using poisson method,Indonesian Journal of Electrical Engineering and Computer Science,V-8,I-2,PP-546-548,Y-2017

9. Ayyappan G., Nalini C., Kumaravel A.,Various approaches of knowledge transfer in academic social network,International Journal of Engineering and Technology,V-,I-,PP-2791-2794,Y-2017

10. Kaliyamurthie, K.P., Sivaraman, K., Ramesh, S. Imposing patient data privacy in wireless medical sensor networks through homomorphic cryptosystems 2016, Journal of Chemical and Pharmaceutical Sciences

11. Kaliyamurthie, K.P., Balasubramanian, P.C.An approach to multi secure to historical malformed documents using integer ripple transfiguration 2016 Journal of Chemical and Pharmaceutical Sciences 9

12. A.Sangeetha,C.Nalini,"Semantic Ranking based on keywords extractions in the web", International Journal of Engineering \& Technology, 7 (2.6) (2018) 290-292

13. S.V.GayathiriDevi,C.Nalini,N.Kumar,"An efficient software verification using multi-layered software verification tool "International Journal of Engineering \& Technology, 7(2.21)2018 454-457

14. C.Nalini,ShwtambariKharabe,"A Comparative Study On Differen Techniques Used For Finger - Vein Authentication", Internationa Journal Of Pure And Applied Mathematics, Volume 116 No. 82017 , 327-333, Issn: 1314-3395

15. M.S. Vivekanandan and Dr. C. Rajabhushanam, "Enabling Privacy Protection and Content Assurance in Geo-Social Networks", International Journal of Innovative Research in Management, Engineering and Technology, Vol 3, Issue 4, pp. 49-55, April 2018. 
16. Dr. C. Rajabhushanam, V. Karthik, and G. Vivek, "Elasticity in Cloud Computing", International Journal of Innovative Research in Management, Engineering and Technology, Vol 3, Issue 4, pp. 104-111, April 2018.

17. K. Rangaswamy and Dr. C. Rajabhushanamc, "CCN-Based Congestion Control Mechanism In Dynamic Networks", International Journal of Innovative Research in Management, Engineering and Technology, Vol 3, Issue 4, pp. 117-119, April 2018.

18. Kavitha, R., Nedunchelian, R., "Domain-specific Search engine optimization using healthcare ontology and a neural network backpropagation approach", 2017, Research Journal of Biotechnology, Special Issue 2:157-166

19. Kavitha, G., Kavitha, R., "An analysis to improve throughput of high-power hubs in mobile ad hoc network" , 2016, Journal of Chemical and Pharmaceutical Sciences, Vol-9, Issue-2: 361-363

20. Kavitha, G., Kavitha, R., "Dipping interference to supplement throughput in MANET", 2016, Journal of Chemical and Pharmaceutical Sciences, Vol-9, Issue-2: 357-360

21. Michael, G., Chandrasekar, A.,'Leader election based malicious detection and response system in MANET using mechanism design approach", Journal of Chemical and Pharmaceutical Sciences(JCPS) Volume 9 Issue 2, April - June 2016

22. Michael, G., Chandrasekar, A.,"Modeling of detection of camouflaging worm using epidemic dynamic model and power spectral density", Journal of Chemical and Pharmaceutical Sciences(JCPS) Volume 9 Issue 2, April - June 2016.

23. Pothumani, S., Sriram, M., Sridhar, J., Arul Selvan, G., Secure mobile agents communication on intranet,Journal of Chemical and Pharmaceutical Sciences, volume 9, Issue 3, Pg No S32-S35, 2016

24. Pothumani, S., Sriram, M., Sridhar, Various schemes for database encryption-a survey, Journal of Chemical and Pharmaceutical Sciences, volume 9, Issue 3, Pg NoS103-S106, 2016

25. Pothumani, S., Sriram, M., Sridhar, A novel economic framework for cloud and grid computing, Journal of Chemical and Pharmaceutical Sciences, volume 9, Issue 3, Pg No S29-S31, 2016

26. Priya, N., Sridhar, J., Sriram, M. "Ecommerce Transaction Security Challenges and Prevention Methods- New Approach" 2016 ,Journal of Chemical and Pharmaceutical Sciences, JCPS Volume 9 Issue 3.page no:S66-S68 .

27. Priya, N.,Sridhar,J.,Sriram, M."Vehicular cloud computing security issues and solutions" Journal of Chemical and Pharmaceutical Sciences(JCPS) Volume 9 Issue 2, April - June 2016

28. Priya, N., Sridhar, J., Sriram, M. "Mobile large data storage security in cloud computing environment-a new approach" JCPS Volume 9 Issue 2. April - June 2016

29. Anuradha.C, Khanna.V, "Improving network performance and security in WSN using decentralized hypothesis testing "Journal of Chemical and Pharmaceutical Sciences(JCPS) Volume 9 Issue 2, April - June 2016

\section{AUTHORS PROFILE}

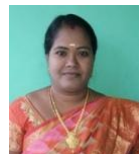

D.Vimala, Assistant Professor, Department of Computer Science \& Engineering, Bharath Institute of Higher Education and Research, Chennai, India

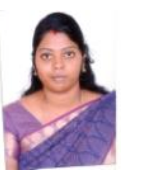

S. Sangeetha, Assistant Professor, Department of Computer Science \& Engineering, Bharath Institute of Higher Education and Research, Chennai, India

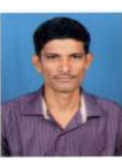

B. Sundar Raj, Assistant Professor, Department of Computer Science \& Engineering, Bharath Institute of Higher Education and Research, Chennai, India 